\title{
Localized states on comb lattices
}

\author{
G. Baldi* \\ Dipartimento di Fisica and INFM, Università di Trento, Via Sommarive 14, 38050 Povo, Trento, Italy \\ R. Burioni and D. Cassi \\ Dipartimento di Fisica and INFM, Università di Parma, Parco Area delle Scienze 7A, 43100 Parma, Italy
}

(Received 7 November 2003; published 28 September 2004)

\begin{abstract}
Complex networks and graphs provide a general description of a great variety of inhomogeneous discrete systems. These range from polymers and biomolecules to complex quantum devices, such as arrays of Josephson junctions, microbridges, and quantum wires. We introduce a technique, based on the analysis of the motion of a random walker, that allows us to determine the density of states of a general local Hamiltonian on a graph, when the potential differs from zero on a finite number of sites. We study in detail the case of the comb lattice and we derive an analytic expression for the elements of the resolvent operator of the Hamiltonian, giving its complete spectrum.
\end{abstract}

DOI: 10.1103/PhysRevE.70.031111

\section{INTRODUCTION}

Recent developments in microelectronics and nanotechnologies have led to the construction of complex structures whose properties are mainly determined by the geometrical arrangement of their elementary components, such as Josephson junctions arrays, quantum dots networks, and molecular devices [1]. This possibility has stimulated the theoretical study of general discrete structures, with particular regard to the influence of topology on physical behaviors.

From a mathematical point of view, a complex structure can be described in terms of an abstract model called a graph, made of sites and links. The sites correspond to the discrete elements of the system and the links represent their mutual relations.

The description of the large-scale topology of complex networks is based on the definition of a parameter, the spectral dimension $\bar{d}$, introduced for fractals by Alexander and Orbach [2] and later rigorously defined on a generic graph by Hattori, Hattori, and Watanabe [3]. The spectral dimension generalizes the Euclidean dimension of regular lattices and rules the universal properties near the critical points and the low temperature thermodynamics [4]. On the other hand, the local structure of a graph can induce many relevant effects. Recent works on Bose-Einstein condensation on inhomogeneous networks have put into evidence an example of a peculiar behavior, due to the geometrical inhomogeneity. A system of noninteracting bosons on a comb lattice, a low dimensional, $\bar{d}=1$, network, presents condensation at finite temperature [5].

Many relevant models on complex networks can be described by an Hamiltonian matrix, composed of a kinetic and a potential term. The former is directly related to the topology of the graph, while the latter takes into account the existence of defects or impurities in the material or the presence of an external field. On regular lattices, the study of the spectral problem for these Hamiltonians is simplified by the

\footnotetext{
*Electronic address: baldi@ science.unitn.it
}

PACS number(s): 05.40.Fb, 63.50.+x, 02.10.Ox

translational invariance. It is well known that in this case the eigenvectors of the Hamiltonian are either localized or extended over the entire lattice.

Here, we will consider the spectral problem for the Hamiltonian operator on a general graph. The absence of translational invariance gives rise to a great variety of eigenstates, which can be localized on particular domains, even of infinite size. A relationship between the localization problem and the properties of a random walker moving on the graph is established and used as a method to solve the spectral problem on an arbitrary network. The effects of the potential matrix on the density of states of the Hamiltonian are investigated and, in particular, the relation between the existence of localized states and the form of the potential is analyzed. The random walk method is then applied to study the effects of the potential on an important case of inhomogeneous network, the comb lattice [6,7]. This is an infinite graph which can be obtained connecting a linear chain, called the finger, to each site of a basic chain, the backbone. The increasing interest in the study of this network is due to the existence in nature of polymers and biological molecules, having the structure of a comb lattice. Moreover, it should be possible to build quantum devices, such as arrays of Josephson junctions [8], microbridges or quantum wires arranged according to the geometry of a comb graph.

The comb graph is characterized by a peculiar continuous spectrum divided in two sections. One corresponds to generalized eigenstates extended on the whole graph. The other presents states localized on the backbone and exponentially decreasing on the fingers of the comb [9]. It is shown that the existence of these states in the high energy region of the continuous spectrum can influence the presence of localized states corresponding to a positive potential. In particular the spectrum of the Hamiltonian is analytically determined in the presence of a potential which differs from zero only on a finite subgraph of the comb lattice.

We begin (Sec. II) with a brief introduction to the mathematical notation of graph theory; we then discuss the definition of the Hamiltonian operator for the vibrational dynamics of a graph and its quantum mechanical behavior; a definition of the random walk problem is also given. Section 
III is devoted to the investigation of the relation between the spectral problem for the Hamiltonian operator and the properties of a suitable random walk. This result is then applied in Sec. IV to the case of the comb lattice, where we analytically determine the density of states of the Hamiltonian using a geometrical-combinatoric technique to compute the random walk generating function. Finally, conclusions are presented in Sec. V.

\section{DEFINITIONS}

\section{A. Graphs}

A graph $\mathrm{G}$ is a countable set $V$ of vertices $i$ connected pairwise by a set $E$ of unoriented links $(i, j)=(j, i)$. Two sites are called nearest neighbors if there is a link between them. The coordination number of site $i$ is the number of links that start from $i$. A path in $\mathrm{G}$ is a sequence of consecutive links $\{(i, k)(k, h) \cdots(n, m)(m, j)\}$. The length of the path is the number of links it contains. A loop is a link between one site and itself. A simple graph is a graph without loops and such that for any two sites $i, j \in V$ there is at most one link between them. A graph is said to be connected if for any two points $i, j \in V$ there is always a path joining them. In the following we will consider connected graphs. The graph topology is algebraically described by its adjacency matrix, which is defined as follows: $A_{i, j}$ is the number of links between $i$ and $j$.

The coordination number of point $i$ can be obtained from $A$ by

$$
z_{i}=2 A_{i, i}+\sum_{j} A_{i, j}
$$

The adjacency matrix is a linear operator defined on the Hilbert space $l^{2}(V)$. One of the most relevant operators in physics is the Laplacian, which, on a graph, is defined [10] by

$$
L=Z-A \text {, }
$$

where $Z$ is the matrix of the coordination numbers: $Z_{i, j}$ $=z_{i} \delta_{i, j}$. This operator is the natural extension to a graph of the usual Laplacian, which acts on the continuous threedimensional space. It has some important spectral properties: its spectrum is real, non-negative and bounded. In particular, on a finite graph, 0 is a simple eigenvalue of $L$ corresponding to the constant eigenvector.

\section{B. Vibrational dynamics and quantum particles on a graph}

The Laplacian operator is directly related to the vibrational dynamics and to the quantum mechanical motion of a particle on the graph. Let us consider $N$ masses $m$ connected by springs of elastic constant $k$. The displacement of the masses from their equilibrium position is described by $N$ vectors $\mathbf{x}_{i} \in \mathbb{R}^{3}$ and, in the approximation of harmonic oscillations, the dynamic is given by the set of equations:

$$
m \frac{d^{2} \mathbf{x}_{i}}{d t^{2}}=-k \sum_{j \sim i}\left(\mathbf{x}_{i}-\mathbf{x}_{j}\right)
$$

where the sum is performed over the nearest neighbors $j$ of the site $i$. Using the adjacency matrix to describe this adja- cency relation and introducing the time Fourier transform $\mathbf{y}(\omega)$ of $\mathbf{x}(t)$, one obtains

$$
\frac{m}{k} \omega^{2} \mathbf{y}_{i}=\sum_{j} L_{i, j} \mathbf{y}_{j}
$$

The problem of finding the normal modes of oscillation is then reduced to the spectral problem for the Laplacian operator.

The quantum mechanical behavior of a particle moving on the graph is described by a discrete Schrödinger equation. The wave function of the particle is a vector $\phi$ belonging to the $l^{2}(V)$ space and the equation is

$$
\sum_{k}\left(\frac{\hbar}{2 m} L_{j, k}+\tilde{U}_{k, k}\right) \phi_{k}=i \hbar \frac{\partial \phi_{j}}{\partial t}
$$

where the Laplacian represents the kinetic term and $\widetilde{U}$ is a diagonal matrix for the potential energy. One can also describe the quantum mechanics on a graph using a tight binding model. This model, in the so-called two center approximation, will again reduce to a Hamiltonian of the form $L$ $+U$.

These examples clearly show the importance of the spectral problem for the operator $L+U$, where $U$ is a diagonal real matrix $U_{i, j}=u_{i} \delta_{i, j}$. In the following we will focus our attention on the study of this problem on a general graph. In this case, it is not possible to apply powerful mathematical tools typical of Euclidean lattices, such as the Fourier transform and the reciprocal lattice. We will here introduce a method, based on random walk techniques, that will enable us to solve the problem on a graph, with a general potential.

\section{Random walks}

The walker is a classical particle moving on a graph G. Starting from a site $i$, at every discrete time step the walker jumps in one of the nearest neighbors of $i$, with probability $p_{i, j}$. The simplest situation (simple random walk) is defined by the probabilities

$$
p_{i, j}=\frac{A_{i, j}}{z_{i}}
$$

It is also possible to introduce traps and sources,

$$
p_{i, j}=\frac{A_{i, j}}{z_{i}+d_{i}}, \quad d_{i} \in \mathbb{R} .
$$

If $d_{i}>0$ the walker can die when it passes through the site $i$, while if $-z_{i}<d_{i}<0$ it is possible that some other particles will be born when the walker passes through the site. The situation with $d_{i}<-z_{i}$ is meaningless because in that case $p_{i, j}$ is negative. One of the interesting quantities in the study of the random walk problem is the probability $P_{i, j}(t)=\left(p^{t}\right)_{i, j}$ that the walker starting from the site $i$ will reach the site $j$ at time $t$. Here, it is more useful to determine another quantity, the so-called generating function of random walks, defined as the discrete Laplace transform of $P(t)$ : 


$$
\tilde{P}(\lambda)=\sum_{t=0}^{\infty} \lambda^{t} P(t) .
$$

These definitions provide us with the basis to study the relationship between the spectral problem for $L+U$ and the random walk problem.

\section{RANDOM WALKS AND LOCALIZATION}

\section{A. The resolvent operator and the generating function}

The spectrum of $H=L+U$ on a graph $\mathrm{G}$ can be determined by studying its resolvent,

$$
R(w)=(H-w)^{-1} .
$$

Here, we will show that it is possible to obtain this operator from the properties of a suitable random walk with traps and sources on the graph G. Remembering the definition (1) we can write the generating function as follows:

$$
\begin{aligned}
\widetilde{P}(\lambda) & =\sum_{t=0}^{\infty}(\lambda p)^{t} \\
& =\left[I-\lambda(Z+D)^{-1} A\right]^{-1} \\
& =\left\{\left[w+\lambda^{-1}(Z+D)-A\right]-w\right\}^{-1} \lambda^{-1}(Z+D),
\end{aligned}
$$

where $D$ is the diagonal matrix, $D_{i, j}=d_{i} \delta_{i, j}$. Choosing the matrix $D$ and the variable $\lambda$ to satisfy the condition,

$$
D+(1-\lambda) Z+\lambda w I-\lambda U=0,
$$

the last term of the previous equation will contain the resolvent operator $R(w)$. In the particular case of a regular graph, with $Z=z I$, it is convenient to satisfy the condition by setting $\lambda=z /(z-w)$ and $D=\lambda U$. In the general situation we can satisfy the condition by setting

$$
\begin{gathered}
\lambda=1, \\
D=U-w I .
\end{gathered}
$$

With these definitions, the probability jump matrix becomes a function of $w$,

$$
p(w)=(Z+U-w I)^{-1} A,
$$

and the generating function $\widetilde{P}(\lambda, w)$ a function of $\lambda$ and $w$. The relation (2) between $\widetilde{P}$ and the resolvent operator $R(w)$ can be written as

$$
R(w)=\widetilde{P}(1, w)(Z+U-w I)^{-1} .
$$

From the knowledge of the resolvent operator one can derive the whole spectrum of $H$. In particular, the pure points spectrum coincides with the values of $w$ where $R(w)$ is not defined. The continuous part of the spectrum corresponds to the points of discontinuity of $R(w)$.

It is necessary to point out some observations about the domain of definition of the operators and about the sign of equality in Eq. (4). The matrix $p$ is a probability jump matrix if the conditions

$$
w \in \mathbb{R} \quad \text { and } z_{i}+U_{i}-w>0 \quad \forall i
$$

are satisfied. The series $\Sigma_{t} p(w)^{t}$ defines the generating function $\widetilde{P}(1, w)$ in its domain of convergence. In many cases we can obtain the generating function using some geometrical and combinatory methods that lead us to an analytic extension of the series outside its domain of convergence and outside the region where $p$ can be considered a probability. It is known that in some cases two analytic extensions of the same function can be different. This problem is easily solved by choosing for $R(w)$ the correct analytic extension of the series, taking into account the fact that the spectrum of $H$ is a subset of $\mathbb{R}$. An example of this situation will be presented in the following sections.

\section{B. Combinatorial methods}

Before analyzing the special case of the comb graph it is useful to introduce a result that can be applied to a general class of networks.

To compute the generating function $\widetilde{P}(\lambda)$, let us consider a random walk with traps and sources, described by the probability jump matrix $p_{i, j}$ introduced in Eq. (1), on a given graph G.

Let us define the quantity $F_{i, j}(t)$ as the probability for the walker to start from site $i$ at time 0 and to arrive in $j$ for the first time at time $t$. The Laplace transform $\widetilde{F}(\lambda)$ of $F(t)$ is related to the generating function $\widetilde{P}(\lambda)$ by [11]

$$
\widetilde{P}_{i, j}(\lambda)=\widetilde{F}_{i, j}(\lambda) \widetilde{P}_{i, i}(\lambda)+\delta_{i, j} .
$$

When the site $i$ does not belong to any closed path, $F_{i, i}$ can be decomposed as follows:

$$
F_{i, i}(t)=\sum_{j} p_{i, j} H_{j}(t-2) p_{j, i}=\frac{1}{z_{i, i}+a} \sum_{j} A_{i, j} H_{j}(t-2) p_{j, i} .
$$

Here $H_{j}(t)$ is the probability that the walker, starting from site $j$, will return on $j$ at time $t$ without passing from $i$ [17] and $a$ is the value of $u_{i}$. Introducing the generating functions and using the relation (5) we obtain

$$
\left(z_{i, i}+a\right)\left[\widetilde{P}_{i, i}(\lambda)\right]^{-1}=a+z_{i, i}\left[\widetilde{P}_{i, i}^{a=0}(\lambda)\right]^{-1},
$$

where $\widetilde{P}_{i, i}^{a=0}(\lambda)$ indicates the generating function when $a=0$.

\section{THE COMB LATTICE}

The comb graph (Fig. 1) is an infinite graph, which can be obtained connecting to each site of a linear chain, called the backbone, another chain called the finger. The sites of the comb can be naturally labelled introducing two integer indices $(x, y)$ with $x, y \in \mathbb{Z}$, where $x$ labels the different fingers and $y$ represents the distance from the backbone.

Let us consider on this graph the spectral problem for the operator $H=L+U$ with $U$ different from zero only on a finite number $N$ of sites. We will call "holes" or "walls" the nonzero elements of $U$.

When the attention is focused on the backbone, one can find, in some particular cases, an analytical mapping between 


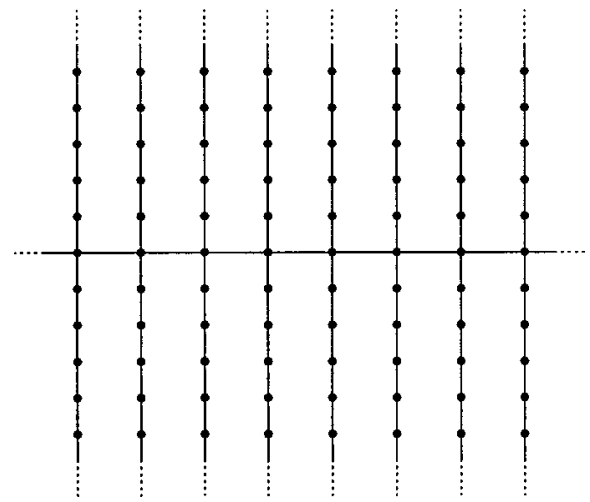

FIG. 1. Two-dimensional comb graph.

the comb lattice and a linear chain with a suitable local potential. This happens, for example, in the situation described in Ref. [12]. If, on the contrary, one aims to determine the eigenstates over the whole comb structure, the random walk technique must be used. This topological method applies to the entire graph without distinctions between backbone and fingers.

The method is based on the analysis of the random walk defined by the jump matrix probability introduced in (3). The idea is to find the generating function for the random walk corresponding to the case with $w=0$, that is a random walk with a finite number $N$ of traps or sources, and then to substitute $z_{(x, y)}$ with $z_{(x, y)}-w$.

In what follows $F$ and $P$ will refer to a comb lattice. A quantity with the superscript 1 refers to the linear chain while subscript 2 means a comb lattice or part of it.

\section{A. The single hole}

We will start our analysis from the case of a potential made by a single hole or wall situated in site $(0, y)$, that is when $u_{\left(x^{\prime}, y^{\prime}\right)}=a \delta_{x^{\prime}, 0} \delta_{y^{\prime}, y}$. Let us call $i$ the site $(0, y)$ and 0 the site $(0,0)$.

Result (6) allows for the calculation of the generating function $\widetilde{P}_{i, i}(\lambda)$ for a simple random walk on the comb graph.

Let us call ${ }^{1} \widetilde{P}_{i, j}^{(x)}(\lambda)$ the generating function of the random walks on a finite and open (the first and last site of the chain are not connected to each other) linear chain made of $x$ sites and where the probability for the walker to jump from one site to the next one is always $\frac{1}{2}$. This function can be calculated using standard methods $[13,14]$. We begin by calculating $F_{i, i}(t)$. Let us call $P_{0,0}^{(i)}(t)$ the probability for the walker to start from 0 and go back to 0 at time $t$ without passing through site $i . F_{i, i}(t)$ can be decomposed in

$$
\begin{aligned}
F_{i, i}(t)= & \frac{1}{z_{b}}{ }^{1} H_{0}(t-2)+\frac{1}{z_{b}}{ }^{1} P_{0,0}^{(y-1)}(t-2)+\sum_{t_{1}, t_{2}, t_{3}}\left[\frac{1}{\left(z_{f}\right)^{3} z_{b}}{ }^{1} P_{0, y-2}^{(y-1)}\right. \\
& \left.\times\left(t_{1}\right) P_{0,0}^{(i)}\left(t_{2}\right)^{1} P_{0, y-2}^{(y-1)}\left(t_{3}\right) \delta_{t_{1}+t_{2}+t_{3}+4, t}\right],
\end{aligned}
$$

where $z_{b}$ is the coordination number of a site of the backbone while $z_{f}$ refers to the fingers. The first term describes the possibility for the walker to move from $i$ in the direction opposite to the backbone. The second term corresponds to the walker moving towards the backbone but without touching it. The last term is the probability for the walker to move towards the backbone, to reach it, to move a time $t_{2}$ around and then to go back to site $i$. Multiplying the two members of this equation by $\lambda^{t}$ and summing over $t$ we obtain for $\widetilde{F}_{i, i}(\lambda)$ the following expression:

$$
\widetilde{F}_{i, i}(\lambda)=\frac{\lambda^{2}}{z_{b}}\left[{ }^{1} \widetilde{H}_{0}(\lambda)+{ }^{1} \widetilde{P}_{0,0}^{(y-1)}(\lambda)\right]+\frac{\lambda^{4}}{z_{b} z_{f}^{3}}\left[{ }^{1} \widetilde{P}_{0, y-2}^{(y-1)}(\lambda)\right]^{2} \widetilde{P}_{0,0}^{(i)}(\lambda) .
$$

The function ${ }^{1} H_{0}(t)$ is the probability of going back to 0 on a half-linear chain, starting from 0 .

The function $\widetilde{P}_{0,0}^{(i)}(\lambda)$ can be computed in an analogous way by decomposing the probability $F_{0,0}^{(i)}(t)$ in terms of the functions ${ }^{1} H_{0}(t)$ and ${ }^{2} H_{0}(t)$ and ${ }^{1} P_{0,0}^{(y-1)}(t)$.

\section{B. A finite number of holes and walls}

This method can be applied to the case of a potential made of $N$ nonzero elements. To do this, we need another ingredient, the generating function ${ }^{2} \widetilde{P}_{(r, 0)(s, 0)}^{(x)}(\lambda)$, representing the probability for the walker to move between two sites of the backbone of a comb graph made by a finite backbone and by $x$ infinite fingers. We can express this function in terms of ${ }^{1} \widetilde{P}_{r, s}^{(x)}(\lambda)$ and of ${ }^{1} \widetilde{P}_{0,0}^{\prime}(\lambda)$, as follows [15]:

$$
{ }^{2} \widetilde{P}_{(r, 0)(s, 0)}^{(x)}(\lambda)={ }^{1} \widetilde{P}_{0,0}^{\prime}(\lambda){ }^{1} \widetilde{P}_{r, s}^{(x)}\left(\frac{\lambda}{z_{f}}{ }^{1} \widetilde{P}_{0,0}^{\prime}(\lambda)\right) .
$$

The function ${ }^{1} \widetilde{P}_{0,0}^{\prime}(\lambda)$ is the generating function of the probability for the walker to go back to site 0 on a linear infinite chain where the probability of escaping from site 0 is $\frac{1}{4}$ instead of $\frac{1}{2}$.

Exploiting the result (6), we calculate $\widetilde{P}_{i, i}(\lambda)$, where $i$ indicates the site of a wall or of a hole, that we can assume located in $(0, y)$, for a random walk problem with $N-1$ traps or sources. $\widetilde{P}_{i, i}(\lambda)$ can be decomposed as in Eqs. (5) and (7) where $\widetilde{P}_{0,0}^{(i)}(\lambda)$ can be expressed in terms of the functions ${ }^{1} \widetilde{H}_{0}$, ${ }^{2} \widetilde{H}_{0},{ }^{1} \widetilde{P}_{r, s}^{(x)},{ }^{2} \widetilde{P}_{(r, 0)(s, 0)}^{(x)}$. This can be done by identifying all the sites that correspond to a wall or to a hole and all the sites where a finger, containing a wall or a hole, intercepts the backbone. We have thus solved the random walk problem for the case with $w=0$. We obtain the function $\widetilde{P}_{i, i}(\lambda, w)$ by substituting $z_{(x, y)\left(x^{\prime}, y^{\prime}\right)}$ with $z_{(x, y)\left(x^{\prime}, y^{\prime}\right)}-w$ and using the result (4) we get an expression for the resolvent operator $R(w)$.

Let us discuss now some problems concerning the domain of definition of these functions. The presence of some complex square roots in the expression of $\widetilde{P}_{i, i}(\lambda, w)$ makes it a multivalued function. However the resolvent $R(w)$ is, for its 


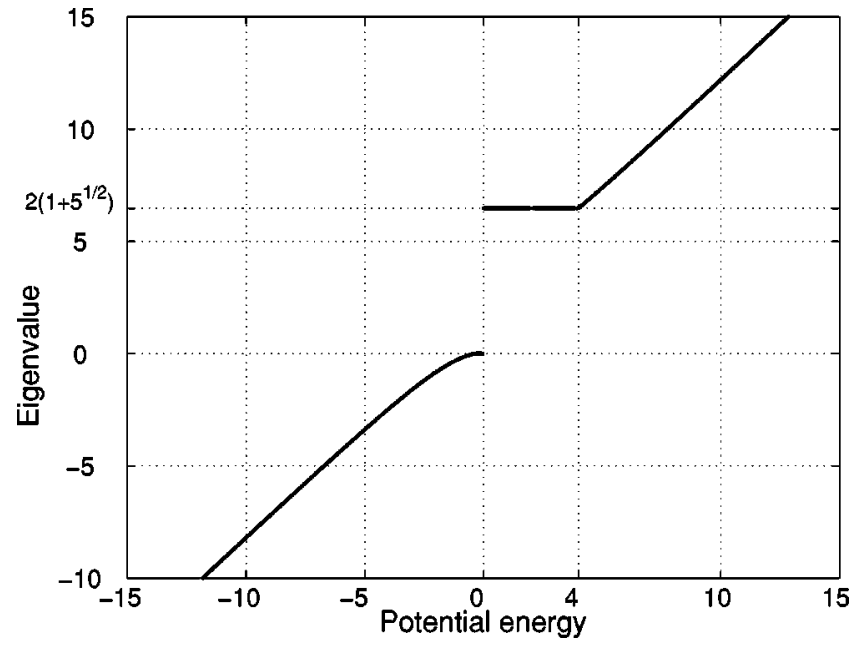

FIG. 2. Eigenvalue as a function of the strength of the potential, which is made of only one wall or hole situated at a distance $y=3$ from the backbone.

nature, a single-valued function, because the spectrum of an operator is univocally defined. In exploiting the result (4) to determine $R(w)$ from $\widetilde{P}_{i, i}(\lambda, w)$ it is therefore necessary to choose the correct branch of the function. This is obtained by considering that $H$ is a self-adjoint operator, so that $R(w)$ can have singularities only on the real axis, and satisfying the following condition:

$$
\widetilde{P}_{i, i}(0, w)=P_{i, i}(0)=1 \quad \forall w .
$$

The knowledge of an analytic expression for some elements of the resolvent operator $R(w)$ leads us to a local analysis of the spectrum of the operator $H$. After calculating the element $R_{i, i}(w)$ of the resolvent, the points of divergence of this function will coincide with elements of the pure points spectrum of $H$. When the potential $U$ is made of only one wall or hole we obtain that $H$ has one and only one proper eigenvalue, which is always outside the continuous part of the spectrum. The continuous part of the spectrum is the same as for the situation with $U=0$ and coincides with the interval $[0,2(1$ $+\sqrt{5})]$. From our analysis we also get the dependence of the eigenvalue as a function of the distance $y$ of the wall or hole from the backbone and of its strength $a$. It is interesting to note that the curve of the eigenvalue as a function of $a$ tends to be constant, as $y$ increases, for values of $a$ in the interval [0,4] (see, for example, Fig. 2). This is rather intriguing, because the value 4 is also the point of separation between the region of the continuous spectrum of measure 1 and the region of so-called measure zero [9].

Let us consider now the case of a potential $U$ made of two nonzero elements. After having computed the function $\widetilde{P}_{i, i}(\lambda, w)$, where $i$ corresponds to a nonzero element of the potential matrix, we come to the following results:

(i) the proper eigenvalues are at most two.

(ii) for some particular values of the variables involved, that is the position of the two walls or holes and their strength, there can be one or even no eigenvalues at all. This can happen when the two walls or holes are close enough and their strength is small.

This result is quite surprising because if there is only one wall or hole there is always one and only one eigenvalue.

\section{DISCUSSION AND CONCLUSIONS}

By analyzing the case of the comb lattice, we have shown how it is possible to analytically determine the spectrum of the Hamiltonian operator on a complex network. When the potential assumes non zero values only on a finite subgraph of the considered graph the solution is reached exploiting the random walk method. The topological nature of the method allows for its application to a vast class of graphs.

The relationship between the motion of the random walker and the spectral problem for the Hamiltonian can also be exploited for numerical computations. The random walker is naturally implemented as a Monte Carlo method, thus allowing the analysis of complex potentials on every finite graph.

The Monte Carlo method consists in the evaluation of the generating function $\widetilde{P}_{i, i}(1, w)$ of a random walker simulated on a computer.

In the case of the comb lattice the number of localized states is always less than or equal to the number of nonzero elements in the potential matrix. It will be of great interest to see if this situation also occurs on other graphs and if it is possible to devise some regularity in the dependence of the number of localized states as a function of number, position and strength of holes and walls.

This analysis is intended to find a general condition for localization on complex networks, in the same spirit of the Anderson's result [16] for regular lattices (notice that the mechanisms inducing localization on graphs can be very different from the ones occurring on a regular geometry). We think that this problem can be fruitfully investigated using the Monte Carlo method, based on the motion of a random walker, to study the localization over a generic graph with a random potential.

On regular lattices the spatial dimension plays a fundamental role since, when the dimension is less than two, a short range disorder is sufficient to induce localization. Heuristic results obtained on several inhomogeneous structures strongly suggest that on graphs the corresponding relevant parameter is the spectral dimension and that short range disorder gives rise to localization for $\bar{d}<2$. It should be noticed that, on regular lattices with $d<2$, one of the main factors inducing localization is the absence, in the graph topology, of closed self-avoiding paths (loops). One could suppose this consideration applies even to graphs, since no loops are present on the comb lattice. However, it can be shown that the spectral dimension can be less than two even in the presence of loops, provided that their number increases slowly enough with their size, as in the case of the Sierpinski gasket.

Besides the investigation of a possible extension of the Anderson's theorem, a future development of the present work will be the study of the relationship between the random walks' properties and the shape of the eigenvectors of the Hamiltonian. 
[1] K. E. Drexler, Nanosystems: Molecular Machinery, Manufactoring and Computation (Wiley Interscience, New York, 1992).

[2] S. Alexander and R. Orbach, J. Phys. (France) Lett. 43, L625 (1982).

[3] K. Hattori, T. Hattori, and H. Watanabe, Prog. Theor. Phys. Suppl. 92, 108 (1987).

[4] R. Burioni and D. Cassi, Phys. Rev. Lett. 76, 1091 (1996).

[5] R. Burioni, D. Cassi, I. Meccoli, M. Rasetti, S. Regina, P. Sodano, and A. Vezzani, Europhys. Lett. 52, 251 (2000).

[6] D. C. Mattis, Phys. Rev. B 20, 349 (1979).

[7] G. Weiss and S. Havlin, Physica A 134, 474 (1986).

[8] E. Simanek, Inhomogeneous Superconductors: Granular and Quantum Effects (Oxford University Press, Oxford, 1994).
[9] R. Burioni, D. Cassi, M. Rasetti, P. Sodano, and A. Vezzani, J. Phys. B 34, 4697 (2001).

[10] W. Woess, Bull. London Math. Soc. 26, 1 (1994).

[11] D. Cassi and S. Regina, Mod. Phys. Lett. B 6, 1397 (1992).

[12] R. Burioni, D. Cassi, I. Meccoli, and S. Regina, Phys. Rev. B 61, 8614 (2000).

[13] E. W. Montroll and G. H. Weiss, J. Math. Phys. 6, 167 (1965).

[14] C. S. O. Yokoi, A. Hernandez-Machado, and L. RamirezPiscina, Phys. Lett. A 145, 82 (1990).

[15] D. Cassi and S. Regina, Phys. Rev. Lett. 76, 2914 (1996).

[16] P. W. Anderson, Phys. Rev. 109, 1492 (1958).

[17] $H$ has only the subscript $j$ because $i$ and $j$ are nearest neighbors in view of the presence of $A_{i, j}$. 